\title{
Trail Making Test
}

National Cancer Institute

\section{Source}

National Cancer Institute. Trail Making Test. NCI Thesaurus. Code C92205.

A neuropsychological test of visual attention and task switching that requires a subject to connect a sequence of 25 consecutive targets. It provides an easy to administer and reliable assessment of attention, sequencing, cognition, visual search, and motor function. 